\title{
Experiences and perspectives on the return of secondary findings among genetic epidemiologists
}

\author{
Catherine M. Stein, $\mathrm{PhD}^{1}$, Roselle Ponsaran, MA², Erika S. Trapl, $\mathrm{PhD}^{1}$ and \\ Aaron J. Goldenberg, PhD, $\mathrm{MPH}^{2}$
}

\begin{abstract}
Purpose: While there has been a recent increase in scholarship around developing policies for the return of results from genetic sequencing, it is not clear whether these approaches are appropriate for genetic epidemiology studies. Because genetic epidemiological research increasingly utilizes genome sequencing methods, particularly in large data sets where researchers did not directly ascertain the subjects, it is important to understand researchers' perspectives on the return of results.
\end{abstract}

Methods: We conducted an online survey of members of the International Genetic Epidemiology Society to document the diversity of experiences and impressions regarding return of results. The survey contained both closed and open-ended questions.

Results: Among our respondents who enroll their own research participants, only $21 \%$ return secondary findings. Most respondents do not search their sequence data for clinically actionable findings not associated with their disease of interest. Many feel that genetic epidemiologists have a unique perspective on the return of results and that research studies should not follow the same procedures as clinical sequencing studies.

Conclusion: Precision medicine initiatives that rely on both clinical and "big data" genomic research should account for variation in researcher perspectives and study design limitations when developing policies and standard practices regarding the return of results.

Genetics in Medicine (2019) 21:1541-1547; https://doi.org/10.1038/s41436018-0369-x

Keywords: genetic epidemiology; return of results; incidental findings; research ethics

\section{INTRODUCTION}

Current advances in molecular genetics have made it possible to interrogate millions of genetic variants at one time. These high-throughput platforms, including exome sequencing (ES) and genome sequencing (GS), allow genetic epidemiologists to conduct genome-wide searches for rare variants associated with disease, as well as identify novel polymorphisms that may be associated with disease risk. These assays also allow investigators to observe known disease-causing polymorphisms that may be clinically actionable as well as others that have been associated with disease risk across many studies but do not yet have clinical implications. It may be in the interest of individuals participating in genetic research to obtain the results of such studies if there are potential implications on their own health. However, recent scholarship on return of results from research studies may not consider the unique situation of genetic epidemiologists, who work with such large data sets and have little, if any, contact with study participants such that return of results may simply not be feasible.

Several groups have developed guidelines for return of secondary findings from clinical genomic sequencing. A
National Heart, Lung, and Blood Institute working group made recommendations for return of secondary findings that meet certain criteria, among them that the result come from a CLIA-certified lab, that the genetic finding be actionable, and that disclosure of such finding(s) has been ethically approved. ${ }^{1}$ Furthermore, several studies have examined experiences and views of researchers on return of results, but these have generally focused primarily on clinical researchers. ${ }^{2-4}$ This is likely due to the fact that clinical researchers have a primary role in obtaining informed consent and collection of clinical data. Kleiderman et al. ${ }^{4}$ included nonclinical researchers in their study, and although it is unclear what type of research these individuals conducted, their respondents reported that the research infrastructure and study design were not in place to return secondary findings, return of research findings should be limited to the original research question, and that consent forms were overly paternalistic and did not accommodate the return of results. Further, they reported that researchers did not have a clinical relationship with research participants, and there is a lack of training in the delivery of genetic information.

${ }^{1}$ Department of Population \& Quantitative Health Sciences, Case Western Reserve University, Cleveland, OH, USA; ${ }^{2}$ Department of Bioethics, Case Western Reserve University, Cleveland, OH, USA. Correspondence: Aaron J. Goldenberg (aaron.goldenberg@case.edu) 
While it may be desirable for some groups of participants to have the results of such studies returned, ${ }^{2,3,5}$ there may be several logistical concerns in large research studies, many of them unique to genetic epidemiologists. The field of genetic epidemiology includes human geneticists, statistical geneticists and biostatisticians, epidemiologists, and population geneticists interested in identifying genes that confer risk for complex traits. Many of their studies are large and population-based. Genetic epidemiologists often work collaboratively with clinicians who ascertain subjects, so that the genetic epidemiologists themselves have little or no contact with the participants. Further, given that this type of research is typically not associated with patients and clinical decisionmaking, genetic epidemiological studies often do not use CLIA-certified laboratories for the generation of research data, yielding secondary findings that do not meet accepted best practices of only releasing clinically valid findings.

Another complexity is that many studies ascertained subjects years or even decades ago, before technologies like ES and GS were imaginable. Thus, the possibility of returning results to study participants was unforeseen, and therefore was not part of the human subjects review process by the institutional review boards (IRBs). In addition, analysis of this ES and GS is incredibly time-intensive because of their size, so identification of incidental findings within these large data sets is often beyond study-specific analyses and requires resources that may be unavailable. Most projects may not involve genetic counselors who could work with patients to explain genetic findings, presenting additional difficulty in appropriately interpreting any secondary findings with study participants.

Lastly, many genetic epidemiologists who are interested in developing statistical and bioinformatic methods for the analysis of large data sets often obtain data from repositories such as dbGaP. Alternatively, data from public repositories may be used for the replication of findings from other data sets. There is little IRB guidance regarding return of results from these types of studies given that researchers would have no contact with the original data collectors or participants and would be unable to return results to the study participants.

The ethical and logistical issues surrounding return of secondary findings are coming to the fore now with increased interest in precision medicine and "big data" analyses. This new frontier in public health genetics represents the collision of the fields of medical genetics and genetic epidemiology, and the success of precision medicine depends on the collaboration of these fields. ${ }^{6}$ Little work has been done to examine the impact of return of results on researchers in the field of genetic epidemiology. Thus, we sought to more fully document the diversity of experiences of genetic epidemiologists in approaching ES and GS data and the potential for return of results. Through this study, we aimed to identify unique practices within genetic epidemiology that may require special attention by policy makers and provide an empirical foundation for promoting the integration of genetic epidemiologists' perspectives into the evolving dialogue over polices and practices surrounding the return of results.

\section{MATERIALS AND METHODS}

To address major issues in return of results, a brief survey was developed by the authors (C.M.S. and A.J.G.) and informed by the members of the Ethical, Legal, and Social Issues (ELSI) Committee of the International Genetic Epidemiology Society (IGES). IGES is an international society of scientists who are largely focused on large population-based studies to identify genetic factors associated with disease. To ensure face validity, ELSI members were engaged in two ways. First, a smaller subcommittee reviewed the overall survey and provided feedback to ensure that the questions were comprehensive to meet the study aims. Next, the survey was sent to the full ELSI Committee for pretesting and additional feedback. Following edits recommended by ELSI, the final survey was administered using Qualtrics to IGES members via email in fall 2014, with the request going out a total of three times. Respondents were entered into a drawing to win one of two \$50 Amazon gift cards. No personal identifying information was collected; email addresses for individuals interested in the drawing were collected and stored separately from survey data.

The survey consisted of 20 questions concerning aspects of their research, whether or not their primary research project returned targeted findings and/or secondary findings, logistics surrounding the return of results, and open-ended questions that solicited general feedback on these topics (survey available in Supplemental Materials). Within the survey, we defined "targeted findings" as those related to the scientific objectives of their study (for example, for a study related to the genetics of colon cancer, there may be genes related to risk for colon cancer specifically). Also within the survey, we defined "incidental findings" as "those findings from either genome-wide association data and/or whole exome/genome sequencing data that may have clinical relevance that were not part of your original research hypotheses and/or specifically related to the medical condition under study." To be consistent with the current state of the field, we refer to "secondary findings" through the rest of this paper.

Analyses included frequencies of categorical responses, and were performed in SPSS version 22. Text from open-ended items was extracted and a thematic analysis was conducted to identify emergent themes. The survey was reviewed and approved by the Case Western Reserve University IRB.

\section{RESULTS}

The survey was sent to all members of IGES. At the time of this study IGES had 311 members. A total of 216 individuals responded to the survey, resulting in a $69 \%$ response rate. These individuals had a variety of roles in their research projects, with most being principal investigator (PI) or coinvestigator $(69 \%)$, and $24.2 \%$ reported being a biostatistician/data analyst (Table 1 ). Their primary research areas varied widely; many reported studying cancer $(24.7 \%)$ or chronic disease $(34.7 \%)$, though a similar proportion focused 
Table 1 Demographic characteristics of respondents Question/response

\begin{tabular}{ll}
\hline Focus area of primary research project & \\
\hline Genetic epidemiology of cancer & 47 \\
& $(24.7)$ \\
\hline $\begin{array}{l}\text { Genetic epidemiology of chronic disease (cardiovascular, } \\
\text { diabetes, eye, autoimmune) }\end{array}$ & 66 \\
\hline Genetic epidemiology of infectious disease & $4(2.1)$ \\
\hline $\begin{array}{l}\text { Genetic epidemiology of psychiatric disease, learning } \\
\text { disorders, and/or communication disorders }\end{array}$ & $16(8.4)$ \\
\hline Rare Mendelian disorders & $3(1.6)$ \\
\hline Statistical genetics/methodology development & 43 \\
\hline Another focus area & $(22.6)$ \\
\hline Role on this project & $11(5.8)$ \\
\hline Principal investigator & 86
\end{tabular}

\section{Coinvestigator}

Biostatistician/data analyst

Study coordinator/manager

Another role

Number of research projects that you work on that have genetic components

$\begin{array}{ll}1 & 12(6.1) \\ 2 & 23 \\ & (11.7)\end{array}$

3

31

4

5 or more

Where were study subjects enrolled —check all that apply

United States 40

North America other than United States

South America

2

Europe 1

Africa 17

Australia

Asia

When were study subjects enrolled-check all that apply

Prior to 1980

1980-1990

13

1991-1995

21

$1996-2000$

29

2001-2006

2006-present

ahen respondents could indicate more than one category, percentages are not provided. Percentages calculated out of number of valid responses (excluding missing data). on statistical genetics/methodology development (22.6\%), implying they do not have a primary clinical area of interest. Most respondents work on five or more research projects. While most respondents enrolled study subjects in the United States, many also enrolled study participants internationally, which is unsurprising because this survey was given to an international society. Only 51 of the 216 respondents indicated their study participants were enrolled after 2006, showing that many genetic epidemiologists' studies enrolled subjects many years before the development of GS technologies that would enable identification of secondary findings within research data.

\section{Quantitative analysis}

We defined "incidental findings" as results from either genome-wide association studies (GWAS) and/or ES/GS data that may have clinical relevance but are not part of the original research question. Of our respondents who enroll their own research participants, only 12 reported that they return any incidental (secondary) findings (Table 2). Many respondents indicated that results cannot be returned because of restrictions by the IRB or because they do not have the appropriate type of genome-wide data (Table 3 and Supplemental Table 1). Because genetic epidemiologists tend to work with very large data sets, we asked how the respondents go about the identification of secondary findings. Most (61\%) responded that they do not search the data for genetic variants that do not have a potential association with the medical condition under study, and an additional $4 \%$ responded that they filter out such genetic variants prior to doing further analysis. While many respondents indicated that their IRBs or data restricted the type of results that could be returned, many indicated that different types of results should be returned, especially clinically validated findings and variants with reproductive implications (Table 3). Lastly, of those respondents who indicated that they analyze secondary data, the responses regarding obligation to return secondary findings varied widely, ranging from no obligation to contacting the PI of the original study.

Importantly, 23\% of respondents indicated that they did not feel that the American College of Medical Genetics and Genomics (ACMG) recommendations on return of secondary findings from clinical sequencing ${ }^{7}$ should apply to genetic epidemiology studies, and 37\% indicated they were not sure. Also, 54\% of respondents indicated that genetic epidemiologists have a different perspective on the approach to secondary findings than medical geneticists and researchers in other genetics fields.

\section{Analysis of open-ended questions}

Participants were asked a series of open-ended questions regarding their perspectives on secondary findings and return of research results from genetic epidemiology studies. 
Table 2 Experiences with incidental findings ${ }^{\mathrm{a}}$ Question/response

$N(\%)^{\mathrm{b}}$

\begin{tabular}{|c|c|}
\hline \multicolumn{2}{|c|}{$\begin{array}{l}\text { Does your consent form distinguish between targeted and } \\
\text { incidental findings? }\end{array}$} \\
\hline Yes & $14(24)$ \\
\hline No & $44(76)$ \\
\hline \multicolumn{2}{|c|}{ Do you return any incidental findings? } \\
\hline Yes & $12(21)$ \\
\hline No & $45(79)$ \\
\hline \multicolumn{2}{|c|}{$\begin{array}{l}\text { Do you follow the same procedures with return of incidental } \\
\text { findings (e.g., validation in a CLIA-certified lab, consultation } \\
\text { with a medical geneticist, etc.) that you use for return of } \\
\text { targeted findings? }\end{array}$} \\
\hline Yes & $10(83)$ \\
\hline No & $2(17)$ \\
\hline
\end{tabular}

What kinds of incidental findings do you return? Check all that apply.

Actionable findings, such as PKU, where a medical

9

intervention exists

Clinically validated findings, such as BRCA1/2, which would

require additional counseling

Variants with reproductive/family planning implications

3

(carrier status)

Variants with no known significance

$P K U$ phenylketonuria.

aThese data are from participants who indicated that they enroll their own research subjects.

${ }^{b}$ When respondents could indicate more than one category, percentages are not provided.

\section{Views on return of results}

Participants were first asked whether they felt that genetic epidemiologists have different perspectives on the return of results than researchers in other areas of genetics. Fifty-four percent of our participants said yes, $12 \%$ said no, and $34 \%$ were unsure. Among participants who believed that genetic epidemiologists have a different perspective, two primary related themes emerged: (1) the inherent distance between researchers and participants in genetic epidemiology and (2) the population-level focus of research questions in genetic epidemiology.

For many respondents, genetic findings are not returned because they do not generally work with patients and are more removed from the clinical world, and therefore should not be specifically searching for clinical actionable findings to return to participants. Without that clinician-patient relationship, genetic epidemiologists do not know what patients would or would not want back, or how patients would want them to handle these results given their lack of training in managing results with clinical significance. One participant stated that "[c]linical geneticists and counselors have a direct relationship with a patient. Clinical people know what the original purpose of the testing was and whether knowing incidental findings would be desired.
Table 3 Researcher perspectives and approach to the identification of incidental findings

Question/response

$N(\%)^{\mathrm{a}}$

As a PI/key personnel on a project that enrolls research

participants, what type of findings do you believe should be

returned, regardless of what your current consent form says?

Check all that apply.

Actionable findings, such as PKU, where a medical

intervention exists

Clinically validated findings, such as BRCA1/2, which would

33

require additional counseling

Variants with reproductive/family planning implications

(carrier status)

Variants with no known significance

2

Other

If for any of your projects, you analyze secondary data (e.g., from a repository, dbGaP, WTCC, and/or through a consortium), what do you believe your obligation is, if any, to return genetic results to study participants?

Contact the PI of the study that enrolled the study

participants, and provide the finding

Nothing — the PI should have already found any important

results

Nothing - I am not covered on the PI's ethical review (IRB), so I 24 (19)

have no legal, moral, or ethical obligation

Not applicable-I only analyze primary data

Other

How do you approach the identification of "incidental

findings" (potentially disease-causing variants with clinical

relevance)?

I intentionally filter out genetic variants not relevant to the

$6(4)$

medical question I am studying

I do not search the data for genetic variants that do not have a potential association with the medical condition I am

studying, but I do not intentionally filter them out

I intentionally search the data for known variants that I believe 9 (5)

have clinical relevance and make a note of them

I intentionally search the data for all known variants regardless

of their clinical relevance and make a note of them

I do not worry about incidental findings or do not have the

level of genetic data (GWAS or ES/GS) to observe them

The ACMG has made formal recommendations on the return of incidental findings: "We recommend that laboratories performing clinical sequencing seek and report mutations of the specified classes or types in the genes..." and provide a list of 56 genes with "known pathogenic" or "expected

pathogenic" sequence variation. Do you think that the results of sequencing conducted in research studies should follow

these same recommendations?

Yes

No

Don't know

Do you feel that genetic epidemiologists have a different perspective and/or approach to incidental findings and return 
Table 3 continued

\begin{tabular}{|c|c|}
\hline Question/response & $N(\%)^{a}$ \\
\hline \multicolumn{2}{|c|}{$\begin{array}{l}\text { of results than researchers/professionals in other areas of } \\
\text { genetics (medical genetics, genetic counseling, molecular } \\
\text { genetics, etc.)? }\end{array}$} \\
\hline Yes & $92(54)$ \\
\hline No & $21(12)$ \\
\hline Don't know & $57(34)$ \\
\hline \multicolumn{2}{|c|}{$\begin{array}{l}\text { Should there be separate policies or professional guidance } \\
\text { regarding incidental findings within genetic epidemiology } \\
\text { studies? Please provide an explanation for your response. }\end{array}$} \\
\hline Yes & $77(45)$ \\
\hline No & $40(24)$ \\
\hline Don't know & $52(31)$ \\
\hline \multicolumn{2}{|c|}{$\begin{array}{l}\text { ACMG American College of Medical Genetics and Genomics, ES exome sequen- } \\
\text { cing, GS genome sequencing, GWAS genome-wide association studies, IRB insti- } \\
\text { tutional review board, PI principal investigator, PKU phenylketonuria, WTCC } \\
\text { Welcome Trust Case-Control Consortium. } \\
\text { aWhen respondents could indicate more than one category, percentages are not } \\
\text { provided. }\end{array}$} \\
\hline
\end{tabular}

A genetic epidemiologist can be somewhat removed from patients. Some studies may use publicly available data (1000 Genomes) and we are quite removed from them. We may not know what the patients originally consented for."

Many participants also felt that genetic epidemiologists have a different approach to return of results and secondary findings because a greater proportion of their research questions focus on population-level data, and are generally not as concerned with individual patient outcomes. These participants also highlighted that in many genetic epidemiology studies findings may be speculative and inconclusive, and therefore inappropriate for returning back to patients:

I think the volume of data we analyze is much larger, in terms of genome-wide coverage, than most other genetics settings where the focus is on a smaller set of candidate genes and/or genes shown to have clinical utility. This presents problems with the practicality of being able to scan for incidental findings....

In contrast, only $12 \%$ of participants believed that genetic epidemiologists do not have a different perspective and indicated that there should be one standard for researchers and clinicians for returning results. A few focused on the importance of patient interest in all genetic research, regardless of the kind of study. One stated that "I think all geneticists are capable, at some level, of evaluating the importance of incidental findings." Interestingly, more than a third (34\%) of participants were not sure if genetic epidemiologists have a different perspective from other researchers.

\section{Polices regarding return of results}

Participants also expressed concerns when asked whether there should be separate policies or professional guidance regarding secondary findings within genetic epidemiology studies. Nearly half (46\%) thought there should, citing concern about the practicality and potential cost of returning results to what could be a very large number of patients. Others also mentioned that they did not have CLIA-approved labs, so they did not think such findings should be reported. One participant cited the need for guidance and clinical collaborators when considering returning results:

If a Gen Epi study is targeting clinically relevant and/or actionable genetic variants, then I think it reasonable to require them to have a clinical collaborator who would be responsible for handling return of results. But absent that, the nonclinical researcher should not provide these results and the consent form should be very clear regarding this policy.

Many participants who were unsure about whether there should be separate policies noted that genetic epidemiology studies might not be comparable with other studies or clinical care. One participant stated "[i]t would be difficult to draw the line between a genetic epidemiology study and another kind of genetic study. This would need to be clarified."

Finally, one researcher further worried about the costs of having to return results to so many participants, stating that "[r]eturn of results using genetic counseling would be astronomically costly for large studies."

\section{Guidance needed regarding return of results for genetic epidemiology studies}

Many respondents were interested in educational opportunities to learn about secondary findings within training programs and for continuing professional education. Others were hopeful for increased guidance from IRBs, governmental agencies, and professional organizations, as well as access to toolkits or other resources to help address issues related to return of results. Finally, many participants expressed a desire for new avenues to pursue cross-disciplinary dialogue among genetic researchers to discuss policies and practices regarding the return of results.

\section{DISCUSSION}

Our findings indicate genetic epidemiologists have a wide variety of experiences and opinions on return of secondary findings to study participants, suggesting that a "one size fits all" approach would not be appropriate. While policies have been developed for clinical research, they often do not apply in a straightforward way to large genetic epidemiological studies for reasons identified in this study. With the advent of "big data," analyses of increasingly large data sets that include thousands of subjects collated through electronic medical records and/or research consortia are becoming the new 
standard in genetic research. Further, ES/GS data are becoming more affordable and are also becoming popular in genetic research. As these types of research facilitate identification of secondary findings, there is an urgent need to develop policy related to the return of secondary findings in such studies.

Our data address an important gap in the literature of addressing perspectives of genetic epidemiologists. A key difference between genetic epidemiology and clinical genetics is the focus on population-based research versus patientcentered investigation. This difference is manifested through the direct connection clinical geneticists have with their patients, versus the lack of connection as mentioned by the genetic epidemiologists in our survey. A similar point was raised by Ramoni et $\mathrm{al}^{8}$ in their recent survey of genetic epidemiologists. However, our survey population differed from Ramoni's in that $27.8 \%$ of our respondents reported recruiting study subjects themselves, and $15 \%$ had at least minimal contact with study subjects, whereas Ramoni's survey population included investigators who obtained dbGaP data and thus were likely not primary data collectors. This central issue is reflective in the current debate over precision medicine and population health. ${ }^{9}$ While precision medicine has become the mantra of genetics in recent years partially due to President Obama's encouragement of the Precision Medicine Initiative, there has been debate whether this is achievable within the population health sciences. As noted by Galea, ${ }^{10,11}$ many diseases of interest are inherently complex, potentially due to a variety of genetic variants with small effects and/or interactions among those genes. The identification of such effects is the mathematical foundation of genetic epidemiology, which necessitates study of very large population samples. Precision medicine works well in diseases like cancer where variants do have large actionable effects, but other chronic and infectious diseases may not fit this model.

Another factor that differentiates most genetic epidemiologists from most medical geneticists is the volume of data being analyzed. Often, genetic epidemiologists analyze hundreds, if not thousands, of subjects at one time. Coupling this with the sheer volume of data from a GWAS/GS/ES study, the identification of secondary findings is no trivial task. This is indicated in our responses; the majority of respondents do not search the data for potential secondary findings, as it would be an added burden to conducting their research. Furthermore, many genetic epidemiologists primarily analyze secondary data and do not have a direct connection to the study participants or the IRB that covered the original study. In a survey of authors of GWAS papers, Ramoni et al. ${ }^{8}$ found that those few individuals who did return results to study participants had direct connections with them.

A second issue is that many genetic epidemiologists work on very long-standing studies (i.e., analysis of data collected years earlier), where the protocols and informed consent forms were developed decades before the advent of genome technologies. At that time, it was impossible to identify secondary findings because studies were either very targeted and/or utilized linkage markers. Any changes to a longstanding study to include uses of new genomic technologies and the possibility of returning individual participant results would require IRB re-examination to assess whether modifications to the core protocol change the perceived risk to participants. An IRB could conclude that the protocol now falls into a different risk category necessitating a different level of review, such as moving from an expedited study to a full board review. Additionally, IRBs would have discretion about whether to allow a protocol addendum or require submission of a new protocol to utilize new technologies or return results. A new review by the IRB might necessitate re-consent of the original study participants, which in many cases might be impossible. Our survey specifically queried about the content of consent forms, which is another facet that was not in Ramoni's study. ${ }^{8}$

Beyond IRB review, investigators running large longitudinal studies will need to consider how to operationalize the return of secondary findings and decide whether there are sufficient resources or appropriate policies to facilitate return of results. Many studies may have insufficient staffing for both analysis and interaction with study participants, and there are concerns that the consent forms or participant educational materials may not have contained enough information to allow for the return of results. For example, there is ongoing debate in the literature as to what consent forms actually cover for these kinds of studies. ${ }^{5,11}$

Additional barriers to returning results in genetic epidemiological research settings as cited by Ramoni et al. ${ }^{8}$ included potentially invalid associations and results not validated by a clinically certified laboratory. Concerns about clinical significance were echoed by our respondents, in addition to the lack of appropriate genetic counseling. Both our study and Ramoni's noted the ethical and logistical challenges with return of results. Yet there is a strong sentiment, both within our data and that of Ramoni, that genetic epidemiologists also do see potential benefit to subjects if results are returned, at least in some circumstances. The issues we have identified shed light on challenges for policy development and should be carefully considered.

A limitation of this study is that respondents were a selfselected convenience sample. Perhaps these investigators had a greater motivation to share their opinions and struggles with return of results. The membership of IGES is not as large as other societies, thereby limiting the sampling frame. In addition, membership of IGES may not be representative of all genetic epidemiologists. While the percentages of respondents indicating a specific experience or opinion may not be reflective of the entirety of IGES, these responses still shed light on an understudied community of genetics researchers. Another potential limitation of this study was our use of the term incidental to describe nontargeted findings from genome sequencing research. This phrase has been debated in recent years, and new language such as secondary or nontargeted findings has become more common. However, at the time our 
survey was fielded, the term incidental was still being utilized within the context of genetic epidemiology, and we believe that the definition we provided to respondents accurately captured the concept of nontargeted results.

\section{Conclusion}

These data illustrate that policies designed for medical genetic research might not always be appropriate for researchers who utilize extremely large data sets to study thousands of subjects at a time. Additionally, while ethical and policy issues surrounding return of results from clinical genetic research have been more fully examined, ${ }^{4,12-14}$ it is not clear whether guidance from these projects are appropriate for larger population-based studies, particularly those where capacity to identify clinically actionable findings was not foreseen at study onset or for data sets where researchers did not directly ascertain subjects.

However, differing views among genetic epidemiologists respondents regarding return of results may not indicate the need for separate policies surrounding population data or GWAS. Rather, we believe these data point to the need for better dialogue between differing fields that utilize genomic technologies in their work to assess differences in research practices and participant interaction. Additionally, there is a need to assess the perspectives of research participants to see if different research uses of their samples and data may impact their own concerns or perspectives on the return of results. Researcher and participant opinion should not be the only drivers of policy or practice changes regarding the return of genomic results. These kinds of data should be considered when deciding if separate policies are needed for genetic epidemiology or whether there may be a way to modify current policies to take these differing research approaches into account. These data may also help determine whether genetic epidemiologists need to change their own practices regarding the return of results given the increased intersection of clinical genomics and population research. As study participants may desire return of results without knowledge of the type of study (e.g., population-based versus patientcentered), further logistical issues need to be considered. Nevertheless, as population health sciences like genetic epidemiology are increasingly used to explore complex genetic influences on disease and health, policies should at least consider the unique study design limitations of large population-based studies when addressing when and how to return incidental findings to research participants.

Finally, these data are timely in light of several institutions initiating large studies aimed at promoting precision medicine. These kinds of initiatives will rely on both the analysis of "big data" and clinical genomic research to develop more targeted medical interventions based on population and individual genomic variation. It is crucial to consider how policies and practices regarding the return of genomic results address the challenges raised by the intersection of clinical and population-based research within precision medicine initiatives.

\section{ELECTRONIC SUPPLEMENTARY MATERIAL}

The online version of this article (https://doi.org/10.1038/s41436018-0369-x) contains supplementary material, which is available to authorized users.

\section{ACKNOWLEDGEMENTS}

We are grateful to Claire Simpson, Gail Jarvik, Diptasri Mandal, and Rob Igo, Jr. for their input on the development of the survey; to the IGES ELSI Committee for their time in testing the survey; and to the leadership of IGES for their support in distributing the survey. Funding for this work was provided by the Center for Genetics, Ethics, Research and Law (CGREAL) (2P50-HG-003390-

06) from the National Human Genome Research Institute.

\section{DISCLOSURE}

The authors declare no conflicts of interest.

\section{REFERENCES}

1. Fabsitz RR, McGuire A, Sharp RR, Puggal M, Beskow LM, Biesecker LG, et al. Ethical and practical guidelines for reporting genetic research results to study participants: updated guidelines from a National Heart, Lung, and Blood Institute working group. Circ Cardiovasc Genet. 2010;3:574-580.

2. Klitzman R, Appelbaum PS, Fyer A, Martinez J, Buquez B, Wynn J, et al. Researchers' views on return of incidental genomic research results: qualitative and quantitative findings. Genet Med. 2013;15:888-895.

3. Wynn J, Martinez J, Duong J, Zhang Y, Phelan J, Fyer A, et al. Association of researcher characteristics with views on return of incidental findings from genomic research. J Genet Couns. 2015;24:833-841.

4. Kleiderman E, Avard D, Besso A, Ali-Khan S, Sauvageau G, Hebert J. Disclosure of incidental findings in cancer genomic research: investigators' perceptions on obligations and barriers. Clin Genet. 2015;88:320-326.

5. Appelbaum PS, Waldman CR, Fyer A, Klitzman R, Parens E, Martinez J, et al. Informed consent for return of incidental findings in genomic research. Genet Med. 2014;16:367-373.

6. Khoury MJ, Gwinn ML, Glasgow RE, Kramer BS. A population approach to precision medicine. Am J Prev Med. 2012;42:639-645.

7. Green RC, Berg JS, Grody WW, Kalia SS, Korf BR, Martin CL, et al. ACMG recommendations for reporting of incidental findings in clinical exome and genome sequencing. Genet Med. 2013;15:565-574.

8. Ramoni RB, McGuire AL, Robinson JO, Morley DS, Plon SE, Joffe S Experiences and attitudes of genome investigators regarding return of individual genetic test results. Genet Med. 2013;15:882-887.

9. Khoury MJ, Galea S. Will precision medicine improve population health? JAMA. 2016;316:1357-1358.

10. Bayer R, Galea S. Public health in the precision-medicine era. N Engl J Med. 2015;373:499-501. 11

11. Pereira S, Oliver Robinson J, McGuire AL. Return of individual genomic research results: what do consent forms tell participants? Eur J Hum Genet. 2016;24:1524-1529.

12. Fossey $R$, Kochan D, Winkler E, Pacyna JE, Olson J, Thibodeau $S$, et al. Ethical considerations related to return of results from genomic medicine projects: The eMERGE Network (Phase III) experience. J Pers Med. 2018;8: pii: E2

13. Jarvik GP, Amendola LM, Berg JS, Brothers K, Clayton EW, Chung W, et al. Return of genomic results to research participants: the floor, the ceiling, and the choices in between. Am J Hum Genet. 2014;94:818-826.

14. Klitzman R, Buquez B, Appelbaum PS, Fyer A, Chung WK. Processes and factors involved in decisions regarding return of incidental genomic findings in research. Genet Med. 2014;16:311-317. 